\title{
Induced pluripotent stem cells in rat models of Parkinson's disease: A systematic review and meta-analysis
}

\author{
YUNXIA ZHANG ${ }^{1,2}$, MEILING GE ${ }^{2}$, QIUKUI HAO ${ }^{2}$ and BIRONG DONG ${ }^{2}$ \\ ${ }^{1}$ Chengdu Medical College, Chengdu, Sichuan 610500; ${ }^{2}$ The Center of Gerontology and Geriatrics, \\ West China Hospital, Sichuan University, Chengdu, Sichuan 610041, P.R. China
}

Received July 18, 2017; Accepted September 21, 2017

DOI: $10.3892 /$ br.2018.1049

\begin{abstract}
The effects of induced pluripotent stem cells (iPSCs) in 6-hydroxydopamine-lesioned rat models of Parkinson's disease (PD) have been evaluated in multiple studies. However, the results evaluating the effectiveness of iPSCs in animal models of PD are mixed, primarily due to their low statistical power. In the current study, a meta-analysis was performed to describe the treatment effect of unsorted iPSCs on behavioral testing in experimental rat models of PD. Databases searched included PubMed, EMBASE, MEDLINE and the Cochrane Library from inception to March 2017. Data were extracted for rotation behavior tests (induced by amphetamine and apomorphine) and limb function tests. A total of eight studies were included in the current meta-analysis, and iPSCs were identified to be efficacious according to the pooled standardized mean differences (SMDs) for improving amphetamine-induced rotational behavior [SMD, -2.16; 95\% confidence interval (95\% CI), -2.93, -1.40; P<0.00001] and apomorphine-induced rotational test (SMD, -1.45; 95\% CI, -2.16, -0.73; $\mathrm{P}<0.0001$ ). The pooled evidence indicated that iPSCs improve rotational behavior in rat models of PD. It was concluded that iPSCs provide a potential approach for developing novel treatment strategies for PD, and the results of this meta-analysis may guide future preclinical and clinical studies.
\end{abstract}

\section{Introduction}

Parkinson's disease (PD) is regarded as the most common neurodegenerative movement disorder, affecting $\sim 1 \%$ of the population aged 65 or older worldwide (1). The key pathological characteristic of PD is the selective loss of dopaminergic neurons in the substantianigra in the midbrain. The current clinical diagnosis of PD is based on the presence of

Correspondence to: Professor Birong Dong, The Center of Gerontology and Geriatrics, West China Hospital, Sichuan University, GuoXue Xiang, Chengdu, Sichuan 610041, P.R. China E-mail: birongdong123@outlook.com

Key words: Parkinson's disease, induced pluripotent stem cells, meta-analysis parkinsonian motor features, such as slowness in movement (bradykinesia) and variable expressions of tremor, rigidity and postural instability. With the aging population and increasing life expectancy in the world, the number of people with PD is expected to increase by $>50 \%$ by 2030 (2). However, to date, dopamine (DA) replacement therapeutic strategies, such as the DA precursor levodopa, only improve the symptoms. However, such therapeutic strategies cannot slow or stop the neurodegenerative process in PD and its therapeutic effect is reduced after 3-5 years of use, which then requires an increased dose and may lead to exacerbated side effects (3). Furthermore, surgical or medical therapeutic strategies that provide superior anti-parkinsonian benefits than levodopa have not been fully investigated. Cell therapy has been reported as a promising strategy for potential neuromodulation in animal models of PD.

In recent years, animal models have been developed, with the most promising research demonstrated in cell therapy that evaluates pathogenesis and treatment effect in PD (4). Neurotoxins or pesticides have been used in animals to kill DA neurons and produce parkinsonian symptoms (4). Fetal ventral midbrain cells $(5,6)$ or embryonic stem cells (ESCs) (7-9), as well as mesenchymal stem cells (MSCs) (4,10-12) have been transplanted into these animal models after in vitro differentiation into neural precursors or DA neurons, and functional recovery has been observed in these studies. However, there are various obstacles in developing these stem cells for treatment: i) Ethical concerns of ESCs; ii) limited tissue supply; iii) the need for immunosuppressive treatment in order to prevent graft rejection and graft versus host disease; iv) graft-induced dyskinesia $(13,14)$; and v) poor improvements for behavioral deficits (15). To avoid these adverse outcomes, it is critical to eliminate the unwanted cells from the donor cell population (16). In addition to fetal ventral midbrain cells, ESCs and MSCs, induced pluripotent stem cells (iPSCs) are another type of cell-based therapeutic strategy. Human adult somatic cells may be reprogrammed to iPSCs in culture, thus providing allogenic dopaminergic neurons and an almost unlimited cell supply for replacement therapy (17). Human iPSCs offer the opportunity to bypass the immune rejection issue associated with allogenic cell transplants and may also diminish the bioethical questions surrounding human embryonic stem cell (hESC) therapeutic strategies (16). 
In order to evaluate the efficiency of iPSCs, PD rat models were lesioned using 6-hydroxydopamine (6-OHDA). Various previous studies have reported that positive results have been observed upon immunohistochemical and behavioral testing. To the best of our knowledge, a systematic review of all these studies in an objective and quantitative manner has never been conducted. Therefore, it is necessary to obtain conclusive evidence to identify whether iPSC treatment is effective in PD models. Therefore, a systematic review and meta-analysis were performed in the present study to evaluate the treatment effect of iPSCs in experimental rat models of PD.

\section{Materials and methods}

Search strategy. All studies reporting the use of iPSCs in PD rat models were identified. The studies were searched based upon the keywords or Medical Subjective Heading $(\mathrm{MeSH})$ terms of 'Parkinson disease, induced pluripotent stem cell(s)'. To ensure a comprehensive systematic search, four databases, PubMed, EMBASE (Ovid, 1974 to 2017 March 31), MEDLINE (Ovid, 1946 to Present), and Cochrane Library (Ovid, Cochrane Central Register of Controlled Trials March 2017) were searched up to March 2017 for all English language publications.

Inclusion and exclusion criteria and data extraction. The inclusion criteria for animal models of PD studies were as follows: i) Object of study: Parkinson's animal model (rat or mice); ii) intervention: Test the effects of unsorted iPSCs on PD in at least one experimental group; iii) comparisons: Sham-controlled group or condition; and iv) outcome: Adequate data on behavioral testing to measure response to treatment. Trials were excluded if any of the following factors were identified: i) Case report, conference abstract, comment, editorial and review; ii) not available in English; and iii) duplicate publications.

Two participants extracted data independently using a predefined data extraction form, with disagreements resolved by careful discussion. The information from each article was collected as follows: First author name and publication year, the source of iPSCs, dose of iPSCs, experimental animal models, including species, number, sex and weight; route of iPSC administration; duration of follow-up period; outcome measures and outcome data from behavioral tests were also included. When reported data for meta-analysis were insufficient or only expressed graphically, data were measured using digital ruler software [engauge digitizer, version 4.1; http://markummitchell.github.io/engauge-digitizer (18)] In addition, attempts were made to contact the study author to inquire about further information. Data presented as means, standard deviation (SD) or standard error of the mean (SEM) were extracted. When only SEM was reported, it was converted to SD for the current meta-analysis.

Quality assessment. The methodological quality of the included studies was evaluated by modifying a previously published 11 -item quality scale $(4,19)$. This modified scale consisted of the following six items: i) Random allocation to group; ii) pretreatment behavioral assessment; iii) blinded assessment of outcome; iv) assessment of $\geq 2$ outcomes;

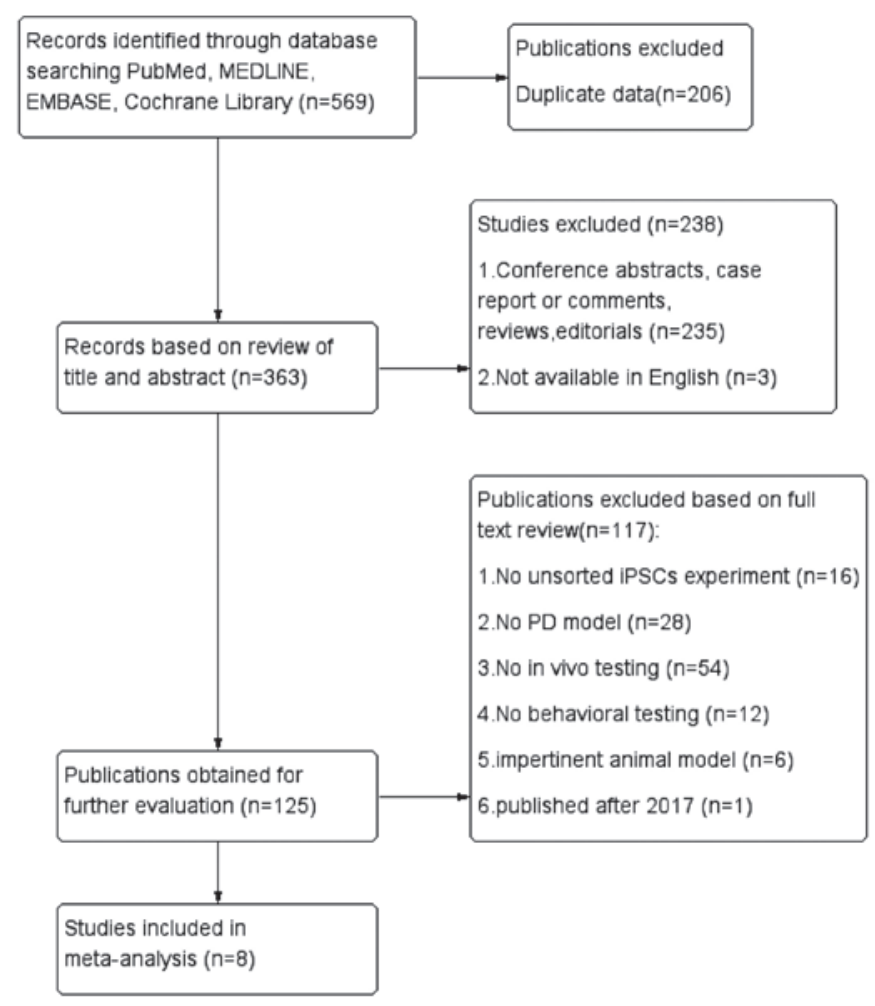

Figure 1. Flow diagram of systematic literature search.

v) compliance with animal welfare regulations; vi) statement of a potential conflict of interest. For the calculation of an aggregate study quality score, one point was attributed for each checklist item reported. Studies were classified into three levels as follows: high risk of bias, $0-2$ points; unclear risk of bias, 3-4 points; low risk of bias, 5-6 points.

Statistical analysis. Outcomes consisted of a rotation behavior test induced by amphetamine or apomorphine and limb function tests, namely cylinder and adjustment stepping tests $(4,16)$. The outcomes were considered as continuous data. Continuous outcomes measured on the same scale were expressed as a mean value and SD, and were analyzed using standard mean differences (SMDs). The $I^{2}$ test was performed to assess the impact of study heterogeneity on the results of the meta-analysis. An $I^{2}$ value $<25 \%$ was considered to below risk of heterogeneity and a fixed-effect model was used for meta-analysis. A value between $25 \%$ and $50 \%$ was regarded as indicative of a moderate level of heterogeneity and $>50 \%$ was considered statistically significant between included studies. For the two, a random-effect model was used to estimate the combined effect sizes. Forest plot was generated to depict the SMD along with its $95 \%$ confidence interval $(95 \% \mathrm{CI}$ ) for each study, as well as the pooled mean difference by combining all studies. Visual inspection of the funnel plot was performed to assess publication bias. Furthermore, sensitivity analysis was conducted by deleting each study individually to evaluate the quality and consistency of the results. All analyses were performed with Review Manager (RevMan; version 5.3, Copenhagen: The Nordic Cochrane Centre, The Cochrane Collaboration, 2014) and $\mathrm{P}<0.05$ was considered to indicate a statistically significant difference. 


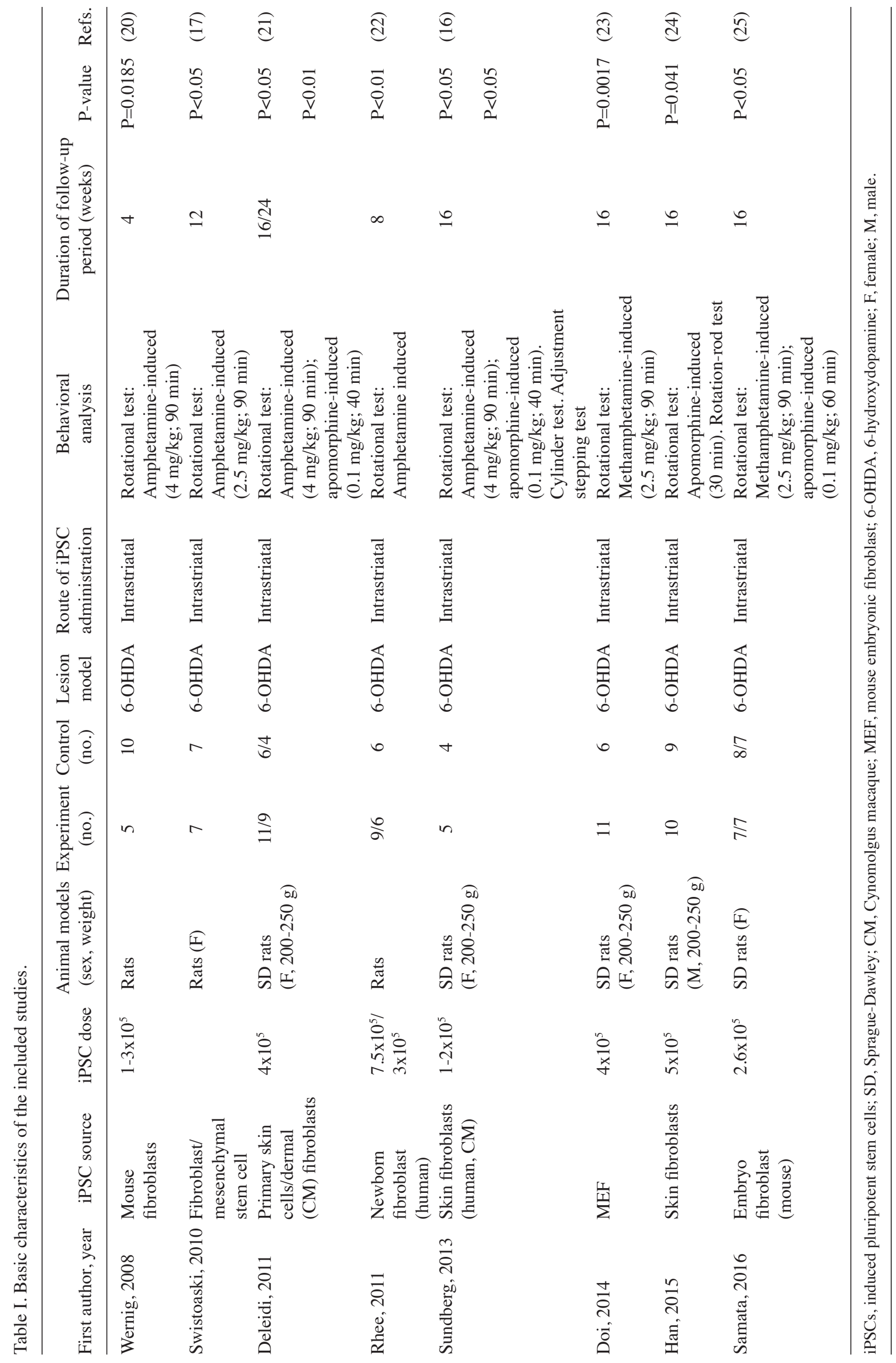


Table II. Risk of bias of included studies.

\begin{tabular}{|c|c|c|c|c|c|c|c|c|}
\hline Author, year & $\mathrm{A}$ & B & $\mathrm{C}$ & $\mathrm{D}$ & $\mathrm{E}$ & $\mathrm{F}$ & Total & Refs. \\
\hline Wernig, 2008 & & & 1 & & 1 & 1 & 3 & $(20)$ \\
\hline Swistoaski, 2010 & & & 1 & & 1 & 1 & 3 & (17) \\
\hline Deleidi, 2011 & & 1 & & 1 & 1 & 1 & 4 & $(21)$ \\
\hline Rhee, 2011 & & & & & 1 & 1 & 2 & (22) \\
\hline Sundberg, 2013 & 1 & 1 & & 1 & 1 & 1 & 5 & (16) \\
\hline Doi, 2014 & & 1 & & & & 1 & 2 & (23) \\
\hline Han, 2015 & & 1 & & 1 & 1 & 1 & 4 & (24) \\
\hline Samata, 2016 & & & 1 & 1 & 1 & 1 & 4 & $(25)$ \\
\hline
\end{tabular}

Studies fulfilling the following criteria: A, random allocation to group; B, pretreatment behavioral assessment; C, blinded assessment of outcome (or computerized); D, assessment of $\geq 2$ outcomes; E, compliance with animal welfare regulations; F, statement of a potential conflict of interest.

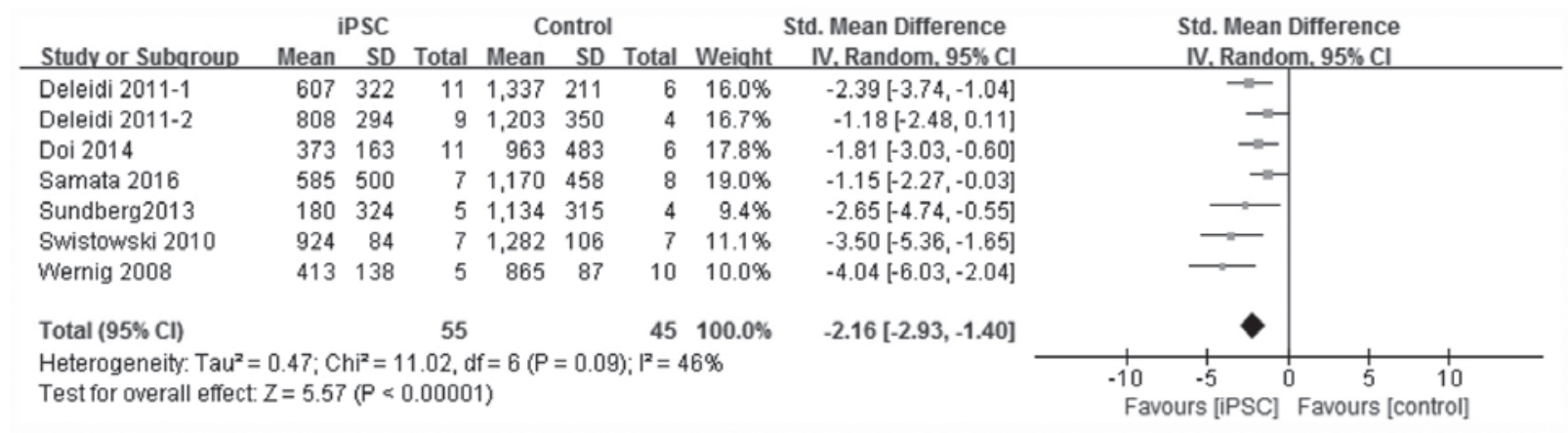

Figure 2. Pooled estimate of amphetamine-induced rotational test with iPSC treatment. IPSC, induced pluripotent stem cells; CI, confidence interval; SD, standard deviation.

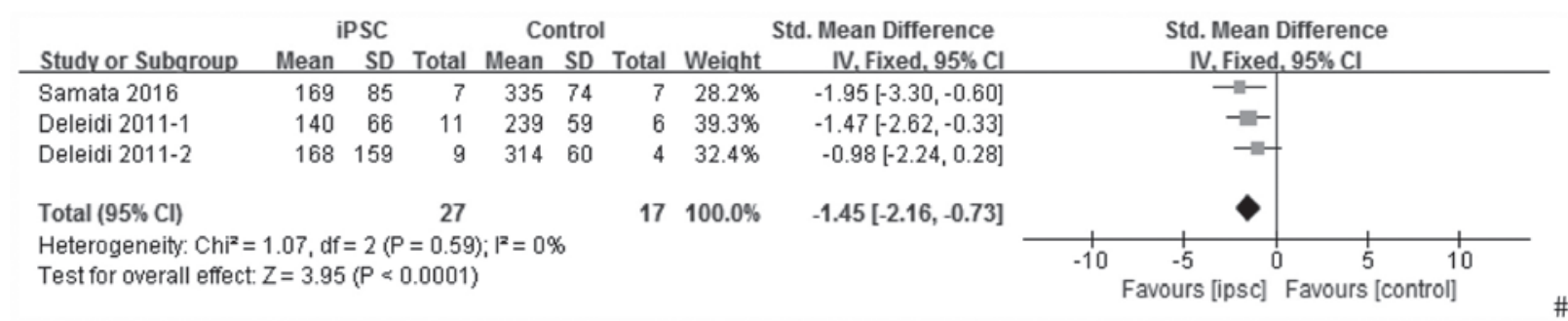

Figure 3. Pooled estimate of apomorphine-induced rotational test with iPSC treatment. iPSC, induced pluripotent stem cells; CI, confidence interval; SD, standard deviation.

\section{Results}

Study inclusion. In total, 569 potentially relevant articles were identified from four databases and 363 records remained after removal of duplicates. After screening the titles and abstracts, 238 papers were excluded for the following reasons: i) Case reports, conference abstracts, comments, reviews and editorials; ii) not available in English. By reading the full text of the remaining 125 articles carefully, 16 studies were excluded due to not having unsorted iPSC experiments; 28 studies were excluded because there was no PD model; 54 were excluded because in vivo testing had not been performed; 12 studies were removed because the animal model was impertinent; and one was published after March 2017. Therefore, eight studies $(16,17,20-25)$ met the eligibility criteria and were included in the meta-analyses. The systematic search to identify all articles for the meta-analysis is presented in Fig. 1.

Study characteristics. A total of eight studies (Table I) described the effects of iPSCs on rat models of PD. The studies that were published between 2008 and 2016, and five out of eight $(62.5 \%)$ studies utilized Sprague-Dawley rats; the other studies did not describe the type of rat used. All the studies used 6-OHDA models and the delivery routes were intrastriatal. The duration of the follow-up period ranged from 4 to 24 weeks. When a study used multiple outcomes or tests and used a separate control group, these separate outcomes or tests were treated as individual experiments. There were 


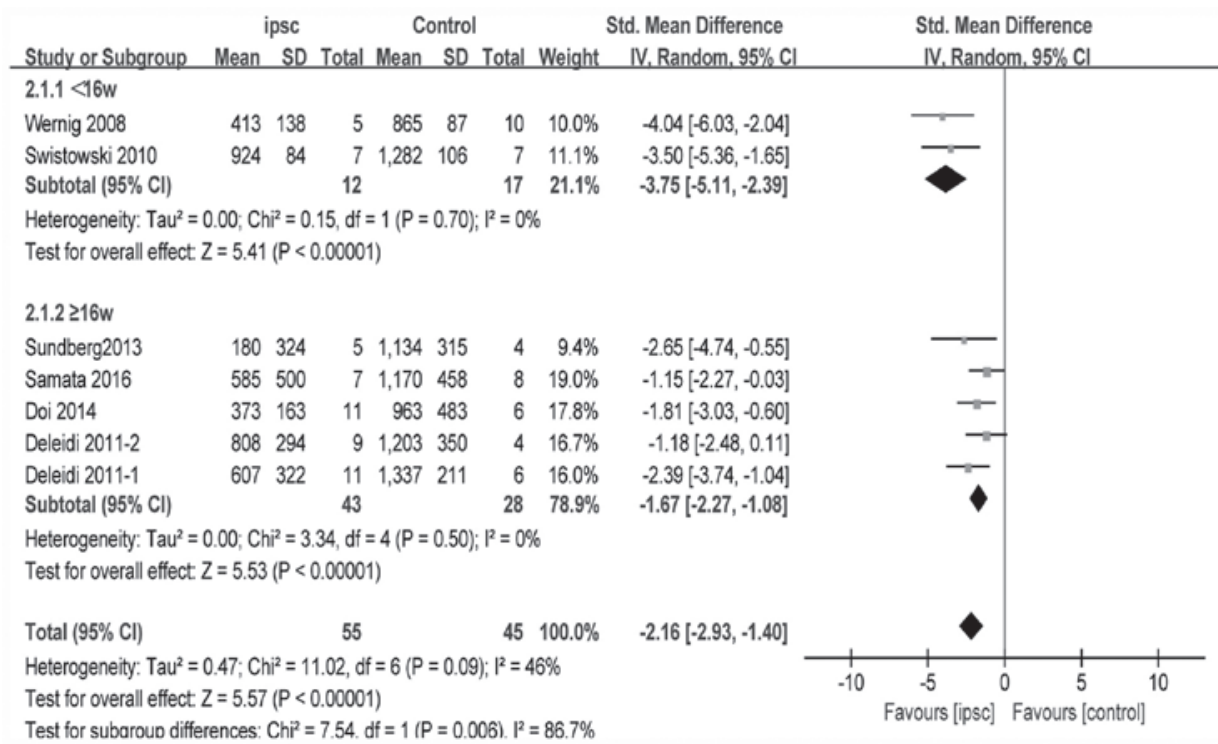

Figure 4. Forest plot of SMD of amphetamine-induced rotational test between iPSC therapy and control groups along with 95\% CI stratified by different durations of follow-up period. SMD, standardized mean differences; CI, confidence interval; SD, standard deviation.

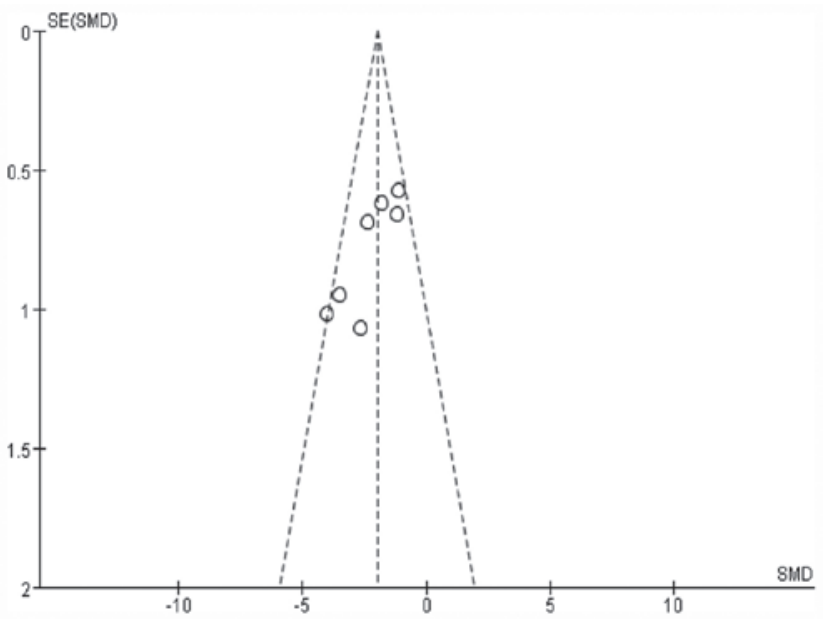

Figure 5. Bias assessment plot for the effect of iPSC on amphetamine-induced rotational test. SMD, standardized mean differences; SE, standard error.

eight experiments conducted to assess amphetamine-induced rotational behavior, five experiments conducted to evaluate apomorphine-induced rotational behavior and only one study evaluated limb function. In addition, there was one study using the rotation-rod test. Certain studies did not include full data in this area, therefore the limb function and rotation-rod test were not included in the meta-analysis.

Quality assessment. The quality assessment of each study is described in Table II. The study quality checklist items that were scored ranged from 2-5 out of a total of 6 points. Two studies got 2 points (25\%); two studies got 3 points $(25 \%)$; and three studies got 4 points (37.5\%); and one study got 5 points (12.5\%). Only one study utilized random allocation of groups. Pretreatment behavioral assessments and blinded assessments of outcomes were described in four and three studies, respectively. Four studies assessed $\geq 2$ outcomes. All studies reported compliance with animal welfare regulations and only one study did not mention the statement of potential conflicts of interest.

Efficacy of iPSCs. All the data for meta-analysis were expressed by diagram, and engauge digitizer 4.1 was used to calculate the means and standard error. For amphetamine-induced rotational tests, data from six studies $(16,17,20,21,23,25)$ were reported. One study (21) used two different iPSC lines, and had separate control group and outcomes. One study (22) reported ratios as outcomes, therefore the required data could not be extracted. The pooled effect size of iPSC therapy was estimated based upon the random-effects model. These experiments reported substantial and significant effects of iPSCs for improving the amphetamine-induced rotational behavior compared with the control group (SMD, -2.16; 95\% CI -2.93 to $-1.40 ; \mathrm{P}<0.00001$; heterogeneity: $\chi^{2}=11.02$, $\mathrm{P}=0.09, I^{2}=46 \%$; Fig. 2). For apomorphine-induced rotation tests, similarly, one study (24) was excluded from the pooled analysis due to the data being represented in the form of a ratio and one study (16) did not describe the outcome in detail; therefore, it was not possible to calculate the mean and SD. Thus only three experiments $(21,25)$ were reported for evaluating the effect of iPSCs on apomorphine-induced rotation tests. The outcome used the fixed-effects model and indicated that iPSCs also improved the apomorphine-induced rotation tests (SMD, $-1.45 ; 95 \% \mathrm{CI},-2.16$ to -0.73 ; $\mathrm{P}<0.00001$; heterogeneity: $\chi^{2}=1.07, \mathrm{P}=0.59, I^{2}=0 \%$; Fig. 3). The forest plot, stratified by different durations of follow-up period, for the amphetamine-induced rotational test is presented in Fig. 4. The pooled effect size in studies following $\geq 16$ weeks and $<16$ weeks with $95 \%$ CI was $-1.67(-2.27,-1.08)$ with $\mathrm{P}<0.00001$ and $-3.75(-5.11,-2.39)$ with $\mathrm{P}<0.00001$, respectively. The heterogeneity of each was $0 \%$, but was $46 \%$ when viewed as a whole.

Sensitivity analysis. The pooled effect size of each study was evaluated for rotation behavior by excluding individual's 
studies sequentially. The results demonstrated that the stability of results significantly changed when the study by Wernig et al (20) was removed from the meta-analysis; the pooled effect size of iPSC therapy with 95\%CI and $I^{2}$ changed to $-1.90(-2.57,-1.23)$ and $26 \%$, respectively (data not shown), which are narrower and lower than the original.

Publication bias. The funnel plot of the studies included in the present meta-analysis for the outcome of amphetamine-induced rotation test is presented in Fig. 5. No evident publication bias was observed through the visual distribution of the funnel plot. The use of a funnel plot was limited for the outcomes of the apomorphine-induced rotation test due to the small number of studies that was evaluated.

\section{Discussion}

In the current study, the efficacy of iPSCs on behavior tests was analyzed in animal models of PD using a meta-analysis to obtain a powerful conclusion. The results demonstrated that iPSCs exert a significant treatment effect on rotation behavior. Behavior tests were regarded as outcomes of this analysis as it is a common parameter to measure functional impairments and recovery in animal models (26) and rotational behavior has also been frequently examined as the measure of functional condition in hemiparkinsonian rodent models (27). In addition, certain studies indicate that limb function is a good indicator of nigrostriatal DA consumption (28). To the best of our knowledge, this is the first meta-analysis providing comprehensive insights into the effect size of unsorted iPSCs in animal models of PD.

In addition to common clinical treatments, such as pharmaceutical drugs and deep brain stimulation, cell replacement therapies have offered a solid foundation for developing an effective therapeutic strategy for PD. The epoch of cell therapy for PD began when Brundin et al (29) transplanted human ventral midbrain tissue into the striatum of PD patients in 1987. Various source tissues have been examined for therapeutic replacement of DA neurons subsequently, such as hESCs, MSCs and DA grafts directly converted from somatic cells. Although numerous studies have demonstrated that those tissues certainly have a significant effect on PD, unfortunately, there were various issues with the studies, such as ethical, technical and practical limitations. Thus, a standardized and limitless cell source for PD is required. Many studies have turned to other appropriate sources, such as iPSCs, which have the potential capacity for self-renewal and are able to differentiate into any somatic cells, including DA neurons (30); therefore, they are ethically more acceptable than certain other stem cell sources. Compared with other stem cells, iPSCs are notable for their powerful pluripotency, infrequent immune rejection and few ethical issues. Furthermore, iPSC-derived neurons capture whole disease genomes without age- and damage-associated epigenetic alterations, which maybe used to model the earliest stages of disease pathogenesis (31). However, there are certain limitations that must be resolved before transplantation of iPSCs, including safety issues associated with tumorigenicity and identifying the mechanisms that enable iPSCs to restore brain function (32).
The results of the present analysis indicate that iPSCs may be a promising candidate for preclinical and clinical trials. All of the included studies used 6-OHDA to induce parkinsonian symptoms rather than other mechanisms, such as 1-methyl-4-phenyl-1,2,3,6-tetrahydropyridine and rotenone, as these promote dopaminergic neuron death with associated motor impairment. Their side effects and lack of specificity are major drawbacks (33). Lesions in the nigrostriatal DA neurons of rats are generated with 6-OHDA, which must be injected into the medial forebrain bundle, substantianigra pars compacta or striatum, rather than systemic administration, to produce the PD model, as it cannot cross the blood-brain barrier (34). The pathological and behavioral phenotypes of this model may differ from the human condition due to differences between species, therefore it is difficult to extrapolate the results obtained from animal models to humans (35). However, the results contribute to understanding the underlying genetic forms of PD and facilitate with establishing disease modifiers and novel targets for possible therapeutic intervention (36).

The evidence that iPSCs may be an effective treatment for PD is encouraging. However, there were certain limitations in the current meta-analysis. First, potential publication bias is likely to exist, despite no evidence of this in the statistical tests, as negative or neutral studies are less likely to be published compared with positive studies. Furthermore, certain outcomes were reported in the form of ratios, therefore could not be included in the current meta-analysis. In addition, behavior tests in animal models of PD cannot fully represent all the components of neurological impairments of PD in humans. The neurological symptoms of PD models consist of motor tests, as well as non-motor deficits. Additionally, there were different sources for each experiment, such as mouse fibroblasts and cynomolgus macaque dermal fibroblasts, and the duration of the follow-up period for rotational tests ranged from 4 to 24 weeks, which may influence the total effect size. Finally, almost all of the included studies had a small sample size (the average number of animals per iPSC and control group was eight and six, respectively). Trials with inadequate sample sizes often run the risk of overestimating intervention effects (37), thus, the current results require careful interpretation.

In conclusion, iPSCs may improve behavioral outcomes in animal models of PD. The current analysis demonstrated that iPSCs provide a potential approach for developing novel treatment strategies for $\mathrm{PD}$, and designing future preclinical and clinical studies.

\section{Acknowledgements}

The authors thank the staff of the Department of Geriatrics Medicine, West China Hospital (Sichuan University, Chengdu, China) for their guidance and support. We also thank Dr Joseph Flaherty, a visiting professor in geriatrics, from Saint Louis University (Saint Louis, MO, USA), for helping with language editing.

\section{Funding}

No funding was received. 


\section{Availability of data and materials}

All data generated or analyzed during the study are included in this published article.

\section{Authors' contributions}

YZ was responsible for formulating the research question, designing the study, collecting the data, screening the papers and quality assessment, and producing the initial draft of the manuscript. MG was responsible for designing the study, screening the studies, collecting the data and statistical analysis. QH was responsible for quality assessment and statistical analysis. BD was responsible for revision of the manuscript.

\section{Ethics approval and consent to participate}

Not applicable.

\section{Consent for publication}

Not applicable.

\section{Competing interests}

The authors declare that they have no competing interests.

\section{References}

1. Kalia LV and Lang AE: Parkinson's disease. Lancet 386 : 896-912, 2015.

2. Dorsey ER, Constantinescu R, Thompson JP, Biglan KM, Holloway RG, Kieburtz K, Marshall FJ, Ravina BM, Schifitto G, Siderowf A, et al: Projected number of people with Parkinson disease in the most populous nations, 2005 through 2030 Neurology 68: 384-386, 2007.

3. Lane EL, Handley OJ, Rosser AE and Dunnett SB: Potential cellular and regenerative approaches for the treatment of Parkinson's disease. Neuropsychiatr Dis Treat 4: 835-845, 2008.

4. Riecke J, Johns KM, Cai C, Vahidy FS, Parsha K, Furr-Stimming E, Schiess M and Savitz SI: A Meta-Analysis of Mesenchymal Stem Cells in Animal Models of Parkinson's Disease. Stem Cells Dev 24: 2082-2090, 2015.

5. Rath A, Klein A, Papazoglou A, Pruszak J, Garcia J, Krause M, Maciaczyk J, Dunnett SB and Nikkhah G: Survival and functional restoration of human fetal ventral mesencephalon following transplantation in a rat model of Parkinson's disease. Cell Transplant 22: 1281-1293, 2013

6. Freed CR, Greene PE, Breeze RE, Tsai WY, DuMouchel W, Kao R, Dillon S, Winfield H, Culver S, Trojanowski JQ, et al: Transplantation of embryonic dopamine neurons for severe Parkinson's disease. N Engl J Med 344: 710-719, 2001.

7. Brederlau A, Correia AS, Anisimov SV, Elmi M, Paul G, Roybon L, Morizane A, Bergquist F, Riebe I, Nannmark U, et al: Transplantation of human embryonic stem cell-derived cells to a rat model of Parkinson's disease: Effect of in vitro differentiation on graft survival and teratoma formation. Stem Cells 24: 1433-1440, 2006.

8. Ambasudhan R, Dolatabadi N, Nutter A, Masliah E, Mckercher SR and Lipton SA: Potential for cell therapy in Parkinson's disease using genetically programmed human embryonic stem cell-derived neural progenitor cells. J Comp Neurol 522: 2845-2856, 2014.

9. Grealish S,DiguetE,KirkebyA,Mattsson B,HeuerA,BramoulleY, Van Camp N, Perrier AL, Hantraye P, Björklund A, et al: Human ESC-derived dopamine neurons show similar preclinical efficacy and potency to fetal neurons when grafted in a rat model of Parkinson's disease. Cell Stem Cell 15: 653-665, 2014.
10. Li Y, Chen J, Wang L, Zhang L, Lu M and Chopp M: Intracerebral transplantation of bone marrow stromal cells in a 1-methyl-4-phenyl-1,2,3,6-tetrahydropyridine mouse model of Parkinson's disease. Neurosci Lett 316: 67-70, 2001.

11. Camp DM, Loeffler DA, Farrah DM, Borneman JN and LeWitt PA: Cellular immune response to intrastriatally implanted allogeneic bone marrow stromal cells in a rat model of Parkinson's disease. J Neuroinflammation 6: 17, 2009.

12. Inden M, Takata K, Nishimura K, Kitamura Y, Ashihara E, Yoshimoto K, Ariga H, Honmou O and Shimohama S: Therapeutic effects of human mesenchymal and hematopoietic stem cells on rotenone-treated parkinsonian mice. J Neurosci Res 91: 62-72, 2013.

13. Carlsson T, Carta M, Winkler C, Björklund A and Kirik D: Serotonin neuron transplants exacerbate L-DOPA-induced dyskinesias in a rat model of Parkinson's disease. J Neurosci 27: 8011-8022, 2007.

14. Politis M, Wu K, Loane C, Quinn NP, Brooks DJ, Rehncrona S, Bjorklund A, Lindvall O and Piccini P: Serotonergic neurons mediate dyskinesia side effects in Parkinson's patients with neural transplants. Sci Transl Med 2: 38ra46, 2010.

15. Olanow CW, Goetz CG, Kordower JH, Stoessl AJ, Sossi V, Brin MF, Shannon KM, Nauert GM, Perl DP, Godbold J, et al: A double-blind controlled trial of bilateral fetal nigral transplantation in Parkinson's disease. Ann Neurol 54: 403-414, 2003.

16. Sundberg M, Bogetofte H, Lawson T, Jansson J, Smith G, Astradsson A, Moore M, Osborn T, Cooper O, Spealman R, et al: Improved cell therapy protocols for Parkinson's disease based on differentiation efficiency and safety of hESC-, hiPSC-, and non-human primate iPSC-derived dopaminergic neurons. Stem Cells 31: 1548-1562, 2013.

17. Swistowski A, Peng J, Liu Q, Mali P, Rao MS, Cheng L and Zeng X: Efficient generation of functional dopaminergic neurons from human induced pluripotent stem cells under defined conditions. Stem Cells 28: 1893-1904, 2010.

18. Mitchell M,Muftakhidinov B,Winchen T, et al: Engauge Digitizer Software. http://markummitchell.github.io/engauge-digitizer. Accessed: May 11, 2017.

19. Schmidt A, Wellmann J, Schilling M, Strecker JK, Sommer C, Schäbitz WR, Diederich K and Minnerup J: Meta-analysis of the efficacy of different training strategies in animal models of ischemic stroke. Stroke 45: 239-247, 2014.

20. Wernig M, Zhao JP, Pruszak J, Hedlund E, Fu D, Soldner F, Broccoli V, Constantine-Paton M, Isacson O and Jaenisch R: Neurons derived from reprogrammed fibroblasts functionally integrate into the fetal brain and improve symptoms of rats with Parkinson's disease. Proc Natl Acad Sci USA 105: 5856-5861, 2008.

21. Deleidi M, Hargus G, Hallett P, Osborn T and Isacson O: Development of histocompatible primate-induced pluripotent stem cells for neural transplantation. Stem Cells 29: 1052-1063, 2011.

22. Rhee YH, Ko JY, Chang MY, Yi SH, Kim D, Kim CH, Shim JW, Jo AY, Kim BW, Lee H, et al: Protein-based human iPS cells efficiently generate functional dopamine neurons and can treat a rat model of Parkinson disease. J Clin Invest 121: 2326-2335, 2011.

23. Doi D, Samata B, Katsukawa M, Kikuchi T, Morizane A, Ono Y, Sekiguchi K, Nakagawa M, Parmar M and Takahashi J: Isolation of human induced pluripotent stem cell-derived dopaminergic progenitors by cell sorting for successful transplantation. Stem Cell Reports 2: 337-350, 2014.

24. Han F, Wang W, Chen B, Chen C, Li S, Lu X, Duan J, Zhang Y, Zhang YA, Guo W, et al: Human induced pluripotent stem cell-derived neurons improve motor asymmetry in a 6-hydroxydopamine-induced rat model of Parkinson's disease. Cytotherapy 17: 665-679, 2015.

25. Samata B, Doi D, Nishimura K, Kikuchi T, Watanabe A, Sakamoto Y, Kakuta J, Ono Y and Takahashi J: Purification of functional human ES and iPSC-derived midbrain dopaminergic progenitors using LRTM1. Nat Commun 7: 13097, 2016.

26. Kirik D, Rosenblad C and Björklund A: Characterization of behavioral and neurodegenerative changes following partial lesions of the nigrostriatal dopamine system induced by intrastriatal 6-hydroxydopamine in the rat. Exp Neurol 152: 259-277, 1998.

27. Cadet JL and Zhu SM; Shu Ming Zhu: The intrastriatal 6-hydroxydopamine model of hemiparkinsonism: Quantitative receptor autoradiographic evidence of correlation between circling behavior and presynaptic as well as postsynaptic nigrostriatal markers in the rat. Brain Res 595: 316-326, 1992. 
28. Emborg ME: Evaluation of animal models of Parkinson's disease for neuroprotective strategies. J Neurosci Methods 139: 121-143, 2004.

29. Brundin P, Strecker RE, Lindvall O, Isacson O, Nilsson OG, Barbin G, Prochiantz A, Forni C, Nieoullon A, Widner $\mathrm{H}$, et al: Intracerebral grafting of dopamine neurons. Experimental basis for clinical trials in patients with Parkinson's disease. Ann N Y Acad Sci 495: 473-496, 1987.

30. Brundin P, Barker RA and Parmar M: Neural grafting in Parkinson's disease Problems and possibilities. Prog Brain Res 184: 265-294, 2010.

31. Jacobs BM: Stemming the hype: What can we learn from iPSC models of Parkinson's disease and how can we learn it? J Parkinsons Dis 4: 15-27, 2014.

32. Xu X, Huang J, Li J, Liu L, Han C, Shen Y, Zhang G, Jiang H, Lin Z, Xiong N, et al: Induced pluripotent stem cells and Parkinson's disease: Modelling and treatment. Cell Prolif 49: $14-26,2016$
33. Valadas JS, Vos M and Verstreken P: Therapeutic strategies in Parkinson's disease: What we have learned from animal models. Ann N Y Acad Sci 1338: 16-37, 2015.

34. Blandini F, Armentero MT and Martignoni E: The 6-hydroxydopamine model: News from the past. Parkinsonism Relat Disord 14 (Suppl 2): S124-S129, 2008.

35. Seibler P, Graziotto J, Jeong H, Simunovic F, Klein C and Krainc D: Mitochondrial Parkin recruitment is impaired in neurons derived from mutant PINK1 induced pluripotent stem cells. J Neurosci 31: 5970-5976, 2011.

36. Li W, Chen S and Li JY: Human induced pluripotent stem cells in Parkinson's disease: A novel cell source of cell therapy and disease modeling. Prog Neurobiol 134: 161-177, 2015.

37. Kjaergard LL, Villumsen J and Gluud C: Reported methodologic quality and discrepancies between large and small randomized trials in meta-analyses. Ann Intern Med 135: 982-989, 2001. 